\section{Morphological, immunophenotypic, and genetic features of chronic lymphocytic leukemia with trisomy 12: a comprehensive review}

\author{
Francesco Autore, ${ }^{1}$ Paolo Strati, ${ }^{2}$ Luca Laurenti ${ }^{1}$ and Alessandra Ferrajoli ${ }^{2}$
}

${ }^{1}$ Hematology Institute, Catholic University of the Sacred Heart, Fondazione Policlinico A. Gemelli, Rome, Italy and ${ }^{2}$ Department of Leukemia, The University of Texas, MD Anderson Cancer Center, Houston, TX, USA

\section{ABSTRACT}

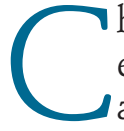
hronic lymphocytic leukemia is an extremely heterogeneous disease and prognostic factors such as chromosomal abnormalities are important predictors of time to first treatment and survival. Trisomy 12 is the second most frequent aberration detected by fluorescence in situ hybridization at the time of diagnosis (10-25\%), and it confers an intermediate prognostic risk, with a median time to first treatment of 33 months and a median overall survival of 114 months. Here, we review the unique morphological, immunophenotypic, and genetic characteristics of patients with chronic lymphocytic leukemia and trisomy 12. These patients carry a significantly higher expression of CD19, CD22, CD20, CD79b, CD24, CD27, CD38, CD49d, sIgM, sIgk, and sIg $\lambda$ and lower expression of CD43 compared with patients with normal karyotype. Circulating cells show increased expression of the integrins CD11b, CD18, CD29, and ITGB7, and of the adhesion molecule CD323. Patients with chronic lymphocytic leukemia and trisomy 12 frequently have unmutated IGHV, ZAP-70 positivity, and closely homologous stereotyped B-cell receptors. They rarely show TP53 mutations but frequently have NOTCH1 mutations, which can be identified in up to $40 \%$ of those with a rapidly progressive clinical course.

\section{Introduction}

Chronic lymphocytic leukemia (CLL), the most prevalent adult leukemia in the Western world, is characterized by the progressive accumulation of mature B cells, and its clinical course is very heterogeneous. ${ }^{1.5}$ Therefore, the identification of prognostic and predictive factors for CLL is critical and this is a field of active investigation. ${ }^{6-12}$ Genetic abnormalities in particular have the potential to serve as prognostic factors in CLL.

Until recently, the low mitotic index of most CLL cells made the use of metaphase cytogenetics difficult, even when metaphases could be generated, because the cellularity was often poor, and abnormalities were detected in only 40 $50 \%$ of cases. Recently, analyzable karyotypes have been obtained in patients with CLL using mitogenic stimulation of CLL specimens with CpG oligonucleotides. ${ }^{13-15}$ Furthermore, the analysis of aberrant chromosomal regions using specific DNA probes through fluorescence in situ hybridization (FISH) has made it possible to detect clonal aberrations in more than $80 \%$ of CLL patients. ${ }^{11,16,17}$

The most common chromosomal abnormalities identified by FISH in CLL are $13 q 14$ deletion (del13q), trisomy $12(+12), 11 q 23.3-q 23.1$ deletion (del11q), and $17 \mathrm{p} 13$ deletion (del17p). ${ }^{11,16,18-22}$ A recently published prospective cohort study of 1494 patients across 199 US centers has shown that del13q is present in approximately $46 \%$ of CLL cases, and +12 is present in $21 \%$ of CLL cases. Del17p and del11q are observed in fewer cases: $12 \%$ and $18 \%$ of CLL cases, respectively. ${ }^{23}$ The frequency of detection of each chromosomal abnormality is influenced by several factors, such as the methods and probes used, the frequency of neoplastic B cells in the specimen analyzed, and the cohort of patients investigated. ${ }^{19,20}$
Haematologica 2018

Volume 103(6):931-938

\section{Correspondence:}

aferrajo@mdanderson.org

Received: January 17, 2018.

Accepted: April 16, 2018.

Pre-published: May 10, 2018.

doi:10.3324/haematol.2017.186684

Check the online version for the most updated information on this article, online supplements, and information on authorship \& disclosures: www.haematologica.org/content/103/6931

\section{(c)2018 Ferrata Storti Foundation}

Material published in Haematologica is covered by copyright. All rights are reserved to the Ferrata Storti Foundation. Use of published material is allowed under the following terms and conditions:

https://creativecommons.org/licenses/by-nc/4.0/legalcode. Copies of published material are allowed for personal or internal use. Sharing published material for non-commercial purposes is subject to the following conditions:

https://creativecommons.org/licenses/by-nc/4.0/legalcode, sect. 3. Reproducing and sharing published material for commercial purposes is not allowed without permission in writing from the publisher. 
Here, we review the unique morphological, immunophenotypic, and genetic characteristics of patients with CLL carrying +12 .

\section{Cytogenetic abnormalities concomitant with +12}

+12 is the second most frequent cytogenetic abnormality identified by FISH in patients with CLL, occurring in $16 \%$ of these patients at the time of initial evaluation. ${ }^{18}$ When identified by FISH, +12 is the sole aberration in approximately $70 \%$ of +12 CLL cases; +12 has been associated with del13q, del11q, or del17p in $18 \%, 8 \%$, and $4 \%$ of +12 CLL cases, respectively. ${ }^{18,19,22,24}$ When identified by chromosome banding analysis, +12 is the sole abnormality in $30 \%$ of +12 CLL cases, but it can be associated with trisomy 18 (5\% of cases) or del14q (3\% of cases); associations with $\mathrm{t}(14 ; 19)(\mathrm{q} 32 ; \mathrm{q} 13)$, trisomy 19, del17p, del13q, and del11q have also been reported. . $^{11,25-27}$

+12 is even more frequent in patients with small lymphocytic leukemia than in those with CLL (28-36\% vs. $13-16 \%) .{ }^{28,29}$ In fact, increased +12 frequency and reduced del13q frequency may represent specific features of small lymphocytic lymphoma with a different prognosis and may distinguish it from classical CLL. ${ }^{28}$

+12 as a single aberration in CLL confers an intermediate prognostic risk, with a median time to first treatment (TTFT) of 33 months and a median overall survival time (OS) of 114 months. ${ }^{18}+12$ that is associated with additional chromosomal abnormalities, including $\mathrm{t}(14 ; 18)(\mathrm{q} 32 ; \mathrm{q} 21)$ and trisomy 18 , portends a poor prognosis independent of NOTCH1 mutation, which is more common in cases with isolated $+12 .{ }^{30}$ Patients with CLL who develop Richter syndrome have a particularly poor prognosis, and +12 is frequent in patients with this syndrome, with an incidence of up to $50 \% .^{31}$ Yi et al. have retrospectively analyzed 330 non-selected CLL patients, using a panel DNA probe to detect cytogenetic abnormalities by FISH, 70 patients were positive for +12 . In patients who carried a favorable or neutral cytogenetic abnormality, including +12 , the co-occurrence of several chromosome abnormalities associated with shorter TTFT and OS. However, in patients with adverse cytogenetic abnormalities, such as del17p or del11q, the co-occurrence of a favorable or neutral clone, including again +12 , portended an improved prognosis. This study challenged the classical hierarchical FISH classification used in CLL, highlighting the prognostic relevance of the intra-tumoral cytogenetic heterogeneity. ${ }^{32}$

+12 is not related to a specific abnormality. However, +12 as a 'driver' mutation can facilitate the appearance of NOTCH1 and FBXW7 mutations: disruptions of FBXW7 function may lead to constitutional activation of NOTCH1 and then to cell proliferation and evasion from apoptosis. The presence of abnormalities of FBXW7 may be implicated in the pathogenesis of the CLL and determine the selection of treatment-resistant clones. ${ }^{33,34}$

\section{Cytopenia in patients with +12 CLL}

Cytopenias are common in patients with +12 CLL. It has been reported that up to $24 \%$ of patients with +12 CLL will develop cytopenias during the course of their disease. ${ }^{35}$ Cytopenias in CLL can result from either bone marrow failure or autoimmune disease. ${ }^{36,37}$ Two studies showed that CLL patients with +12 have a higher incidence of autoimmune cytopenias than infiltrative cytopenias. ${ }^{36,37}$

\section{Morphological features of patients with +12 CLL}

Atypical morphology, defined as the presence of cleaved nuclei and/or lymphoplasmacytoid features in more than $15 \%$ of cells, can be observed in up to $20 \%$ of patients with CLL. ${ }^{38,39}$ Several studies have revealed that +12 is the most common cytogenetic abnormality in these cases. ${ }^{38}$

Athanasiadou et al. evaluated the presence of atypical and typical CLL morphology in a population of $100 \mathrm{CLL}$ patients. ${ }^{38}$ In the +12 subgroup, 17 of the 23 cases analyzed $(74 \%)$ were considered morphologically atypical. Atypical morphology was also frequent in the del11q/del17p subgroup (5 of 7 cases, 71\%). All del13q cases and 45 of 51 cases ( $88 \%$ ) with normal karyotypes had typical morphology. ${ }^{38}$

Ruptured B cells, or smudge cells, are commonly seen in blood smears of patients with CLL. ${ }^{40}$ Levels of smudge cells have been correlated with low CD45 expression on CLL cells by flow cytometry. ${ }^{41}$ Smudge cells are believed to reflect intrinsic CLL cell fragility and cytoskeletal abnormalities, linked to reduced expression of vimentin, and some authors have proposed using percentages of smudge cells to stage CLL. ${ }^{42}$ In patients with +12 CLL, fewer smudge cells are observed than in patients with CLL overall, along with normal levels of CD45 expression. ${ }^{43}$

\section{Immunophenotype of patients with +12 CLL}

Chronic lymphocytic leukemia patients show an immunophenotypic panel that is typically $\mathrm{CD}^{+}, \mathrm{CD}_{2} 3^{+}$, FMC7, surface immunoglobulin (sIg)dim, CD22dim; and CD79bdim: Quijano et al. ${ }^{19}$ established the presence of an atypical immunophenotypic pattern in some subgroups of 180 CLL patients by using combinations of monoclonal antibodies. The atypical immunophenotypic pattern was characterized by a distinct and unusual pattern of expression regarding CD22, CD20, CD79d, FMC7, sIgM, sIgj, sIgk, and CD38. Cases with +12 (23\% of the series), compared with cases with normal karyotypes (38\%), showed significantly higher expression of CD19, CD22, CD20, CD79b, CD24, CD27, CD38, sIgM, sIgk, and sIg $\lambda$ and lower expression of CD43. Among various cytogenetic subgroups, the atypical immunophenotypic pattern identified in overall CLL patients was more frequent in the +12 and del17p subgroups and less frequent in the del13q subgroup; the del11q subgroup presented an equal distribution of the typical and atypical patterns. ${ }^{19}$

An atypical immunophenotype can be defined using the Matutes score, ${ }^{39}$ which is calculated from the following cell surface markers: positive expression of CD5 and CD23, negative expression of FMC7, and weak expression of CD22 and sIg. A modification of the scoring system, called the modified Matutes score, replaces CD22 with CD79b, and this score is considered atypical if less than $4 .^{39}$ Interestingly, with the modified Matutes score, +12 defines a subgroup of CLL with more frequent atypical morphology than with the original scoring system. ${ }^{39,44}$

\section{CD38}

CD38 expression ${ }^{45}$ is significantly more frequent in the +12 and del11q/del17p subgroups of CLL patients (10 of 26 cases and 5 of 10 cases, respectively) than in patients with normal karyotype or in the del13q subgroup of CLL patients (10 of 72 cases and 1 of 5 cases, respectively). ${ }^{38}$ High CD38 expression on CLL cells has been used as a 
surrogate marker of unmutated IGHV genes. ${ }^{46}$ When the threshold for CD38 positivity was set at the standard $30 \%$, higher expression of CD38 was not associated with a significantly impaired TTFT, but a threshold of $40 \%$ for CD38 expression retained its prognostic value for TTFT $(P=0.008)$; so the threshold of CD38 positivity may be raised to $40 \%$ in +12 CLL cases to preserve its prognostic value. ${ }^{46}$ CD38 inhibitors may have particular efficacy in this cytogenetic subtype..$^{47}$ CD $38^{48-50}$ also showed higher expression among CLL cases with both +12 and del11 $q,{ }^{19}$ which could explain the adverse prognosis of this subgroup compared to CLL patients with $+12 .^{50-52}$

\section{ZAP-70}

The negative prognostic impact of $Z A P-70$ expression and the association of $Z A P-70$ with unmutated $I G H V$ are maintained in patients with +12 CLL. ${ }^{46,53,54}$

\section{CD20}

CLL patients with +12 have higher expression of CD20 than CLL patients with del17p, del11q, del13q, or negative FISH results; this expression of CD20 may predict a better response to rituximab-based regimens. ${ }^{55}$

\section{FMC7}

FMC7, an epitope on the CD20 molecule itself, is significantly $(P=0.035)$ more frequent in the +12 subgroup of CLL $(32.0 \%)$ than in the del13q CLL subgroup, normal patients, and the del11q/del17p CLL subgroup (in which $20.0 \%, 11.1 \%$, and $10.0 \%$ were FMC7 positive, respectively). ${ }^{38}$

\section{CD49}

CD $49 \mathrm{~d}$ is expressed in a vast majority of +12 CLL cases and emerged as a negative prognostic factor..$^{56-58}$ Using a $30 \%$ cut off, an analysis was performed on 1200 CLL patients: ${ }^{59} 735$ cases $(61.2 \%)$ were CD49d; whereas 465 cases $(38.8 \%)$ were CD49 $\mathrm{d}^{+}$. A significantly $(P<0.001)$ higher percentage of cases were CD $49 \mathrm{~d}^{+}(89.4 \%)$ in the +12 subgroup than in the other cytogenetic subgroups. Among the CD49d $\mathrm{d}^{+}$CLL patients, those with +12 expressed CD49d at higher mean fluorescence intensity (MFI) levels [median MFI 2200; 95\% confidence interval (CI) 1810-2546] compared with patients without +12 (median MFI 1386; 95\% CI: 1050-1673; P<0.001). CD49d overexpression in +12 CLL is associated with ITGA4 hypomethylation, as shown by quantitative real-time polymerase chain reaction analysis in 74 CLL patients (31 patients who were CD49d and lacked +12 , and 43 patients who were $\mathrm{CD} 49 \mathrm{~d}^{+}$and had +12 ). Patients with +12 who were CD49 $\mathrm{d}^{+}$showed a shorter TTFT than that of patients with +12 who were CD $49 \mathrm{~d}^{-}(P<0.001) \cdot{ }^{56} \mathrm{~A}$ specific tropism of +12 CLL cells toward lymph nodes has been confirmed by the higher proportion of +12 CLL cells in lymph nodes than in peripheral blood or bone marrow. ${ }^{60}$ CD $49 \mathrm{~d}$ overexpression may provide a molecular basis for the peculiar biological behavior of +12 CLL and may predict the development of additional cytogenetic lesions. ${ }^{61}$

\section{CD23}

CD23, a low-affinity receptor for IgE, is expressed on B cells and on monocytes and eosinophils. ${ }^{62}$ CD23 expression varies in CLL: ${ }^{63}$ low CD23 expression, which was correlated with the presence of a prolymphocyte infiltrate in the bone marrow, a higher white blood cell count, and an advanced stage of disease, was reported to be a negative prognostic factor for CLL. ${ }^{64}$ There are two isotypes of CD23: CD23a, whose expression is higher than that of $\mathrm{CD} 23 \mathrm{~b}$, has a role in survival, while CD23b enhances proliferation. ${ }^{65}$ CLL patients can be divided into two groups: one group expressing both isotypes of CD23 at a high level and CD20, CD22, and CD38 at low levels, and one group expressing both CD23 isotypes at a low level, most of them carrying $+12 .{ }^{66}$ Therefore, a high $\mathrm{CD} 23 \mathrm{a} / \mathrm{CD} 23 \mathrm{~b}$ ratio and low CD23 expression combined with high CD20 and CD38 expression may be a useful tool in predicting +12 in CLL and could serve as a marker for a high likelihood of +12 .

\section{Integrins}

Circulating +12 CLL cells show increased expression of the integrins CD11b, CD18, CD29, and ITGB7, and of the adhesion molecule CD323 compared with other CLL cytogenetic groups. These changes are modulated by NOTCH1 mutation status; NOTCH1-mutated +12 cases have lower expression of CD11a, CD11b, and CD18 than wild-type cases. +12 CLL cells also have upregulation of signaling pathways that increase ligand binding and enhance VLA-4-directed adhesion and motility. ${ }^{46}$ Also, increased expression of the $\alpha$-integrins CD11a and CD11b was associated with a shortened TTFT $(P=0.002$ for CD11a; $P=0.027$ for CD11b). The presence of NOTCH1 mutations in the context of +12 was associated with decreased CLL cell expression of CD11a, CD11b, and CD18 to levels similar to those of CLL cells without +12 . Notably, the presence of a NOTCH1 mutation had no impact on CD29, CD49d, or ITGB7 expression on CLL cells. The observed heterogeneity of expression of $\beta_{2}$ integrins in +12 CLL cases can be explained largely by the presence of NOTCH1 mutations. ${ }^{46}$ The expression of the $\beta_{2}$-integrins CD11a/CD18 (LFA-1) and CD11b/CD18 (Mac-1) is down-regulated by the co-existence of NOTCH1 mutations, indicating a novel interaction that may be of importance in aggressive, high-risk CLL. ${ }^{46}$

\section{Adhesion molecules}

Other cell surface markers with a possible prognostic role in +12 CLL are CD25 (also called activation-associated interleukin-2 receptor), which has a role in B-cell proliferation; CD54 (i.e. intercellular adhesion molecule-1), which has a role in cell adhesion and in the homing process; and CD95 (i.e. FasR), which has a role in regulation of apoptosis in lymphoid tissues. Patients with levels of $30 \%$ or higher of CD25+ B-CLL cells had a shorter median TTFT $(P=0.01) .{ }^{67}$ Low CD54 expression was associated with a prolonged median TTFT $(P=0.004)$, low leukocyte count $(P<0.05)$, and low serum lactate dehydrogenase level $(P=0.03)$. CD54 could be a prognostic marker for $\mathrm{CLL}^{68,69}$ and might be a determiner of whether patients present with localized or widespread lymphadenopathies. ${ }^{70}$ High CD95 expression has been correlated with elevated serum lactate dehydrogenase level $(P=0.02)$ and lymphadenopathy $(P=0.02)$. Hjalmar et al. correlated these markers on $\mathrm{CD}^{+} 9^{+}$cells with the occurrence of +12 and atypical lymphocyte morphology. ${ }^{71}$ They found that the mean percentage of $\mathrm{CD} 25^{+} \mathrm{B}$ cells in CLL with atypical morphology and in patients with +12 was lower than that in CLL with typical morphology $(P<0.02)$ and in patients with disomic tumor cells 
$(P<0.03)$. Published data regarding the association between CD25 and +12 report conflicting findings. ${ }^{72-74}$

\section{Stereotyped B-cell receptor prevalence in patients with +12 CLL}

Recurrent 'stereotyped' patterns, ${ }^{75,76}$ known as subsets, have been reported in +12 CLL patients, including a higher prevalence of the IGHV 4-39 gene, particularly in patients who developed Richter Syndrome. ${ }^{77-79}$ Falisi et al. highlighted the fact that $44 \%$ of +12 CLL patients carried stereotyped B-cell receptor ${ }^{33}$ and found a significantly higher prevalence of stereotyped IGHV configurations in +12 CLL patients than in controls $(27 \% ; P=0.01)$. The most prevalent stereotyped subset was subset \#1, followed by subsets \#8, \#10, \#28 and \#59. The most frequently observed IGHV gene was V4-39*01, followed by V1-3*01. IGHV 4-39*01 was the most common gene in +12 CLL patients in the literature, confirming the strong association between +12 , IGHV 4-39*01 stereotype, and Richter syndrome..$^{77-80}$

\section{Immunoglobulin heavy chain mutational status in patients with +12 CLL}

Chronic lymphocytic leukemia subgroups with del11q/del17p and +12 showed greater percentages of unmutated $I G H V$ cases $(80.0 \%$ and $53.8 \%$, respectively) than those with del13q or with normal karyotype (37.5\% and $31.5 \%$, respectively). ${ }^{38}$ Cytogenetic subgroups also showed differences in the IGHV repertoire: IGHV1 genes were observed more frequently in the +12 subgroup $(26.9 \%)$ than in other subgroups $(20.0 \%$ in del11q/del17p and in del13q subgroups and $12.3 \%$ in the normal karyotype). IGHV4 genes were noted at similar frequencies in the +12 subgroup and in the normal group $(34.6 \%$ and $30.1 \%$, respectively), and they were not identified in any cases in the del11q/del17p subgroup. ${ }^{38}$

\section{Molecular abnormalities in patients with +12 CLL}

Chronic lymphocytic leukemia patients with +12 or normal karyotype at diagnosis have an approximately 2fold higher probability of developing poor-risk genetic lesions and entering the intermediate- or high-risk subgroups associated with shorter survival, compared with low-risk CLL patients harboring del13q only. ${ }^{12}$

Chronic lymphocytic leukemia patients with +12 rarely show TP53 mutations or acquire them over time. .2,81,82 on the contrary, NOTCH1 mutations can be identified in 30$40 \%$ of CLL patients carrying $+12 .{ }^{33-86}$ NOTCH1 is a human gene encoding a single-pass transmembrane protein, whose signaling network regulates interactions between physically adjacent cells. Following the pivotal study that identified NOTCH1 mutations in CLL and provided initial evidence on the unfavorable clinical outcome associated with NOTCH1 alterations, ${ }^{87}$ two additional independent studies of the CLL coding genome have identified activating mutations of NOTCH1 gene in approximately $10 \%$ of CLL at diagnosis. ${ }^{88,89}$ The biological contribution of NOTCH1 mutations in determining the aggressive behavior of CLL could be due to the over-representation of genes involved in the cell cycle and proliferation. In at least four series, NOTCH1 mutations had an adverse impact on outcome independently of other clinico-biological features. ${ }^{87-90}$ In an analysis by Del Giudice et al. ${ }^{91}$ NOTCH1 mutations clustered within 104 untreated +12 CLL patients accounted for $24 \%$ of those cases, and were frequently detected in cases with unfavorable biological markers, associated with a particular gene expression profile predicting a poor outcome. NOTCH1 mutations are not associated with TP53 disruption or del11q; this mutual exclusivity could be useful in a genetic hierarchical prognostic model. ${ }^{91}$ NOTCH1 mutations were represented in all cases included by frameshift deletions, in particular c.7544_7545delCT in $88 \%$ of cases, and were preferentially associated with unmutated IGHV $(84 \%$ of cases; $P=0.003) .{ }^{91}$ NOTCH1 mutations were not associated with sex or ZAP-70 positivity (mutated NOTCH1 cases were $62.5 \%$ ZAP- $70^{+}$, and wild-type NOTCH1 cases were $44.2 \%$ ZAP- $70^{+} ; P=0.116$ ). NOTCH1 mutations have been reported at CLL diagnosis in approximately $20 \%$ of chemoresistant cases. ${ }^{33}$ NOTCH1 mutations conferred a higher cumulative probability of Richter syndrome..$^{88,2,93}$ The impact of NOTCH1 mutations on prognosis is less relevant in the presence of concurrent chromosomal aberrations, as worse outcomes are observed among patients with +12 associated with additional chromosomal abnormalities irrespective of NOTCH1 mutation status. ${ }^{30}$

Landau et al. have published the results of a large retrospective cohort study using whole-exome sequencing to analyze the frequency of somatic mutation and copy number abnormalities in 160 patients with CLL. They found that favorable alterations, such as MYD88 mutation, del13q and +12 , represented early clonal drivers of disease; instead, more unfavorable alterations, such as ATM, TP53 and SF3B1 mutations, were subclonal during the early stages of disease, and became clonal at time of first treatment or treatment failure. The same group has more recently published prospective genetic data collected from 278 patients with CLL; while confirming previously published retrospective data, this study found also an association between +12 and mutations in BIRC 3 and $B C O R$. Interestingly, neither $B I R C 3$ nor $B C O R$ mutation was associated with worse survival, in contrast to what had previously been published in smaller studies. . $^{34,35}$

Maura et al. studied the association of CLL with IGF1R expression. ${ }^{94,95}$ IGF1R-IGF1/2 interaction is involved in intracellular cell signaling pathways such as RAS/RAF/MEK/ERK kinases and PI3K-Akt. IGF1R, which is implicated in the activation of the PI3K/Akt and MAPK pathways, was generally over-expressed in CLL cells compared with healthy B cells. ${ }^{96}$ IGF1R expression was strongly associated with unfavorable/intermediate-risk cytogenetic features, in particular +12 , independently from unmutated IGHV, NOTCH1 mutation, or clinical monoclonal B-cell lymphocytosis status. Thus, IGF1R represents a potential novel candidate for specific targeted therapy in +12 CLL patients. In a prospective series, a group of +12 patients, showing low IGF1R expression, mutated IGHV, and wild-type NOTCH1 status had an indolent clinical course, whereas the others, characterized by unmutated IGHV and/or high CD38, mutated NOTCH1, and high IGF1R, had a more aggressive evolution and shorter TTFT. Thus, IGF1R may have a role in discriminating aggressive CLL from intermediate-risk or favorable CLL in the presence of $+12 . .^{95}$

The overexpression of ATF5 in CLL was significantly $(P<0.001)$ associated with chromosomal abnormalities including del11q and $+12 .{ }^{97}$ ATF5 is known for its role in the regulation of cell cycle progression and of differentiation and apoptosis. ATF5 overexpression was most com- 
Table 1. Clinical and laboratory characteristics at diagnosis of patients with +12 chronic lymphocytic leukemia.

\begin{tabular}{|c|c|c|c|c|}
\hline Characteristic & $\begin{array}{l}\text { González-Gascón y } \\
\text { Marín }(n=289)^{82}\end{array}$ & $\begin{array}{c}\text { Strati } \\
(n=250)^{25}\end{array}$ & $\begin{array}{l}\text { Del Giudice } \\
(n=104)^{91}\end{array}$ & $\begin{array}{c}\text { Falisi } \\
(n=44)^{33}\end{array}$ \\
\hline Age (range), years & $68(22-88)$ & $60(32-87)$ & $65(56-72)$ & $62.8(45-86)$ \\
\hline Male sex & $61.6 \%$ & $58.8 \%$ & $54(52 \%)$ & $32(73 \%)$ \\
\hline Binet stage A & $71.1 \%$ & $71 \%$ & & \\
\hline Binet stage B & $23.6 \%$ & $13 \%$ & $36 \%$ Binet $\mathrm{B}$ to $\mathrm{C}$ & \\
\hline Binet stage $\mathrm{C}$ & $5.4 \%$ & $16 \%$ & $36 \%$ Binet $\mathrm{B}$ to $\mathrm{C}$ & \\
\hline Median white blood cell count, $x 10^{9} / \mathrm{L}$ & $19(4.5-294.2)$ & $18(2-681)$ & & \\
\hline Absolute lymphocyte count, $>30^{*} 10^{\circ} / \mathrm{L}$ & $16.8 \%$ & $26.0 \%$ & & \\
\hline Splenomegaly & $15.5 \%$ & $16.0 \%$ & & \\
\hline Hepatomegaly & $5.7 \%$ & $6.0 \%$ & & \\
\hline High $\beta_{2}$ microglobulin & $28.9 \%{ }^{\mathrm{a}}$ & $18.1 \%^{\mathrm{b}}$ & & \\
\hline Unmutated IGHV & $53.8 \%$ & $55.1 \%$ & $58.0 \%$ & $70.6 \%$ \\
\hline CD38 positivity & $37.4 \%$ & $55.0 \%$ & & \\
\hline ZAP-70 positivity & $55.8 \%$ & $45.1 \%$ & & \\
\hline+12 and del11q & $3.9 \%$ & $0 \%$ & $8.0 \%$ & $4.5 \%$ \\
\hline+12 and del17p & $6.1 \%$ & $0 \%$ & $5.0 \%$ & $2.3 \%$ \\
\hline+12 and del13q & $17.0 \%$ & $17.6 \%$ & $18.0 \%$ & $9.1 \%$ \\
\hline
\end{tabular}

${ }^{a} \mathrm{High} \beta_{2}$ microglobulin level defined as above normal range. ${ }^{\circ} \mathrm{High} \beta_{2}$ microglobulin level defined as $\geq 4 \mathrm{mg} / \mathrm{L}$.

mon in the del11q subgroup and second most common in the +12 subgroup, which had poor outcomes with the second shortest TTFT. ATF5 may be a key gene responsible for inducing cell proliferation in CLL patients, and it may explain the mechanisms of disease progression due to cytogenetic abnormalities in these patients. ${ }^{97}$

\section{TTFT and $0 S$ in patients with +12 CLL}

Few reports detail the clinical outcomes of CLL cases with +12 . Characteristics of the patients in these main series are summarized in Table 1. ${ }^{25,33,82,91}$ Here, we review data from the two largest series of patients with +12 CLL published so far. Outcomes from these studies are summarized in Table 2.

A multicenter analysis by González-Gascón y Marín et al. included 2561 patients diagnosed with CLL from 25 Spanish institutions, of whom 289 (11.3\%) patients showed +12 at diagnosis; the analysis excluded patients with monoclonal B-cell lymphocytosis, patients who acquired +12 as clonal evolution, and patients with inadequate follow up. ${ }^{82}+12$ patients had a median TTFT of 42 months (95\%CI: 34-49 months) and a median OS of 129 months (95\%CI: 100-158 months). The findings in this series are similar to those of previous studies that included a lower frequency of +12 cases, approximately 15 $20 \%$, with a median TTFT of 32 months and a median OS of 111 months. ${ }^{18}$ These patients were a median 68 years old, were predominantly male, and had a high frequency of low lymphocyte counts (83\%), low $\beta_{2}$ microglobulin levels $(71 \%)$, early Binet stage $(71 \%)$, low levels of CD38 (63\%), and an absence of significant organomegaly.

González-Gascón y Marín et al. ${ }^{82}$ correlated the proportion of +12 CLL cells with prognosis and identified $60 \%$ as the cut-off value associated with survival. One hundred and seventy-four patients $(60.2 \%)$ carried +12 in less than $60 \%$ of cells, whereas 115 patients $(39.8 \%)$ carried
+12 in $60 \%$ or more of cells. The patients with +12 in $60 \%$ or more of cells had more marked leukocytosis $(P=0.001)$, higher lymphocyte counts $(P=0.006)$, higher levels of serum lactate dehydrogenase $(P=0.03)$, more pronounced splenomegaly $(P=0.001)$, and more advanced Binet stage $(P=0.04)$. In the cohort of $289+12$ patients, $175(60.6 \%)$ required treatment initiation. The patients with +12 in less than $60 \%$ of cells had a lower rate of treatment initiation and longer TTFT $(51.2 \%$ with a median TTFT of 49 months) than did patients with +12 in $60 \%$ or more of cells $75.7 \%$ with a median TTFT of 30 months; $P<0.001)$ in both univariate and multivariate analyses. At the time of analysis, 69 patients $(23.9 \%)$ had died, and there was a significant difference in frequency of deaths $(P=0.009)$ between patients with less than $60 \%$ +12 cells ( $16.7 \%$ with a median OS of 159 months) and patients with $60 \%$ or more +12 cells $(29.6 \%$ with a median OS of 96 months). On multivariate analysis, only Binet stage $(P=0.04)$, del11q in addition to $+12(P=0.01)$, and high $\beta_{2}$ microglobulin levels $(P=0.03)$ were significantly associated with shorter OS. TTFT and OS were shorter in +12 and del11q patients compared with those with +12 as a unique aberration ( 23 months vs. 44 months, $P=0.02$ for TTFT; 44 months vs. 159 months, $P=0.02$ for OS), but no difference could be found in TTFT or OS when +12 was accompanied by del17p or del13q.

Strati et al. analyzed a single-center experience of 250 untreated CLL patients with +12 followed for nine years and compared their outcomes with those of 516 treatment-naive CLL patients who lacked common recurrent abnormalities on FISH analysis (negative for del11q, del13q, del17p, and +12 ), evaluated at the same institution during the same period. ${ }^{25}$ On multivariate analysis, factors significantly associated with +12 were a platelet count of less than $100 \times 10^{9} / \mathrm{L}$ [odds ratio (OR) 2.4; $P=0.03$ ], positivity for $\mathrm{CD} 38$ (OR 2.4; $P=0.001$ ), and a Matutes score of less than 4 (OR 2.4; $P<0.001)$. At a median follow 
Table 2. Clinical outcomes of patients with +12 chronic lymphocytic leukemia.

\begin{tabular}{lcc} 
Outcome & $\begin{array}{c}\text { González-Gascón y Marín } \\
(\mathbf{n}=289)^{82}\end{array}$ & $\begin{array}{c}\text { Stratí } \\
(\mathbf{n}=250)^{25}\end{array}$ \\
Median follow up (range), months & 41 & 51 \\
Treatments & $(1-197)$ & $(1-105)$ \\
Median TTFT (95\% CI), months & $61.0 \%$ & $57.0 \%$ \\
\hline Deaths & 42 & 38 \\
Median OS (95\% CI), months & $21.8 \%$ & $(27-48)$ \\
& 129 & $7.0 \%$ \\
\hline
\end{tabular}

CI: Confidence Interval;TTFT: time to first treatment; OS: overall survival.

up of 51 months (range 1-105 months), 142 patients $(56.8 \%)$ required initiation of treatment. The median TTFT was 38 months (95\%CI: $27-48$ months), significantly shorter than that of FISH-negative patients (82 months; $P<0.001)$. The multivariable model for TTFT showed that a Rai stage of III-IV [Hazard Ratio (HR) 3.3; $P=0.02]$, palpable splenomegaly ( $\mathrm{HR} 2.3 ; P=0.007$ ), and chromosome banding analysis positivity for del14q (HR 3.5; $P=0.004)$ were independently associated with TTFT. The estimated median OS was not reached, as only 18 patients died, and did not differ significantly between patients with or without +12 by FISH $(P=0.22)$.

Followed over time, 6 of these patients $(2.4 \%)$ developed histologically confirmed Richter syndrome after a median ten months (95\% CI: 5-15 months) from diagnosis. This incidence was significantly higher in treated than in untreated patients $(4 \%$ vs. $0 \% ; P=0.04)$ and in patients with +10 compared with the control cohort of patients with normal karyotype $(2.0 \%$ vs. $0.4 \% ; P=0.02)$. Twentytwo patients $(8.8 \%)$ developed a malignancy other than Richter syndrome after a median of 19 months (95\%CI: 6-32 months) from diagnosis. Among second neoplasms,
5 were hematologic malignancies, and 17 were nonhematologic malignancies. In the multivariable model, an absolute lymphocyte count of more than $30 \times 10^{9} / \mathrm{L}$ (HR $14.5 ; P=0.04)$ and development of a second malignant neoplasm (HR 23.8; $P=0.002$ ) remained independently associated with OS. A correlation between CLL and second malignant neoplasms has been reported, but the mechanism linking +12 to the onset of second neoplasms remains unknown. ${ }^{98}$ Eighteen patients $(7.2 \%)$ died during the observation time, 15 of whom had received treatment for CLL. Seven patients died of CLL-related $(n=4)$ or Richter syndrome-related $(n=3)$ causes; 6 patients died of second neoplasms, and 5 died of unrelated causes. There was no significant difference in distribution of the causes of death between treated and untreated patients $(P=0.24)$. The mortality of patients with +12 was only partly related to complications of CLL; the leading causes of death were Richter syndrome and second malignant neoplasms. However, given the evidence of a worse outcome for second malignant neoplasms in CLL, increased surveillance of patients in this specific group should be considered. ${ }^{99}$

\section{Conclusions}

The unique morphology, immunophenotype, and cytogenetics of +12 CLL easily distinguish it from other CLL cytogenetic subtypes. +12 in CLL confers an intermediate prognostic risk, characterized by a median TTFT of 33 months and a median OS of 114 months. Genomic alterations, especially NOTCH1 mutations, can portend a worse prognosis and an increased risk of Richter syndrome.

\section{Acknowledgments}

The authors would like to thank Sarah Bronson, ELS Department of Scientific Publications The University of Texas MD Anderson Cancer Center for editorial assistance.

\section{References}

1. Ghia P, Ferreri AM, Caligaris-Cappio F. Chronic lymphocytic leukemia. Crit Rev Oncol Hematol. 2007;64(3):234-246.

2. Thurmes P, Call T, Slager S, et al. Comorbid conditions and survival in unselected, newly diagnosed patients with chronic lymphocytic leukemia. Leuk Lymphoma. 2008;49(1):49-56

3. Hallek M, Cheson BD, Catovsky D, et al. A report from the International Workshop on Chronic Lymphocytic Leukemia updating the National Cancer Institute-Working Group 1996 guidelines. Blood. 2008;111(12): 5446-5456.

4. Zenz T, Mertens D, Kuppers R, Döhner H, Stilgenbauer S. From pathogenesis to treatment of chronic lymphocytic leukaemia. Nat Rev Cancer. 2010;10(1):37-50.

5. Eichhorst B, Dreyling M, Robak T, Montserrat E, Hallek M; ESMO Guidelines Working Group. Chronic lymphocytic leukemia: ESMO Clinical Practice
Guidelines for diagnosis, treatment and follow-up. Ann Oncol. 2011;22 Suppl 6:vi5054.

6. Rai KR, Sawitsky A, Cronkite EP, Chanana $\mathrm{AD}$, Levy RN, Pasternack BS. Clinical staging of chronic lymphocytic leukemia. Blood. 1975;46(2):219-234.

7. Binet JL, Auquier A, Dighiero G, et al. A new prognostic classification of chronic lymphocytic leukemia derived from a multivariate survival analysis. Cancer. 1981;48(1):198-206.

8. Zenz T, Fröhling S, Mertens D, Döhner H, Stilgenbauer S. Moving from prognostic to predictive factors in chronic lymphocytic leukaemia (CLL). Best Pract Res Clin Haematol. 2010;23(1):71-84.

9. Furman RR. Prognostic markers and stratification of chronic lymphocytic leukemia. Hematology Am Soc Hematol Educ Program. 2010; 2010:77-81.

10. Pospisilova S, Gonzalez D, Malcikova J, et al. ERIC recommendations on TP53 mutation analysis in chronic lymphocytic leukemia. Leukemia. 2012;26(7):1458-1461.
11. Haferlach C, Dicker F, Schnittger S, Kern W, Haferlach T. Comprehensive genetic characterization of CLL: a study on 506 cases analysed with chromosome banding analysis, interphase FISH, $\operatorname{IgV}(\mathrm{H})$ status and immunophenotyping. Leukemia. 2007;21 (12):2442-2451.

12. Rossi D, Rasi S, Spina V, et al. Integrated mutational and cytogenetic analysis identifies new prognostic subgroups in chronic lymphocytic leukemia. Blood. 2013;121 (8):1403-1412.

13. Dubuc AM, Davids MS, Pulluqi M, et al. FISHing in the dark: How the combination of FISH and conventional karyotyping improves the diagnostic yield in CpG-stimulated chronic lymphocytic leukemia. Am J Hematol. 2016;91(10):978-983.

14. Thompson PA, O'Brien SM, Wierda WG, et al. Complex karyotype is a stronger predictor than del(17p) for an inferior outcome in relapsed or refractory chronic lymphocytic leukemia patients treated with ibrutinibbased regimens. Cancer. 2015;121(20):36123621 
15. Jaglowski SM, Ruppert AS, Heerema NA, et al. Complex karyotype predicts for inferior out- comes following reduced-intensity conditioning allogeneic transplant for chronic lymphocytic leukaemia. Br J Haematol. 2012;159(1):82-87.

16. Buhmann R, Kurzeder C, Rehklau J, et al CD40L stimulation enhances the ability of conventional metaphase cytogenetics to detect chromosome aberrations in B-cell chronic lymphocytic leukaemia cells. Br J Haematol. 2002;118(4):968-975.

17. Dicker F, Schnittger S, Haferlach T, Kern W, Schoch C. Immunostimulatory oligonucleotide-induced metaphase cytogenetics detect chromosomal aberrations in $80 \%$ of CLL patients: a study of 132 CLL cases with correlation to FISH, IgVH status, and CD38 expression. Blood. 2006;108(9):3152-3160.

18. Döhner H, Stilgenbauer S, Benner A, et al. Genomic aberrations and survival in chronic lymphocytic leukemia. N Engl J Med. 2000;343(26):1910-1916.

19. Quijano S, Lopez A, Rasillo A, et al. Impact of trisomy 12, $\operatorname{del}(13 q)$, $\operatorname{del}(17 p)$, and del(11q) on the immunophenotype, DNA ploidy status, and proliferative rate of leukemic B-cells in chronic lymphocytic leukemia. Cytometry B Clin Cytom. 2008;74(3):139-149.

20. Stilgenbauer S, Dohner K, Bentz M, Lichter P, Dohner H. Molecular cytogenetic analysis of B-cell chronic lymphocytic leukemia. Ann Hematol. 1998;76(3-4):101-110.

21. Cuneo A, Bigoni R, Rigolin GM, et al. Late appearance of the 11q22.3-23.1 deletion involving the ATM locus in B-cell chronic lymphocytic leukemia and related disorders. Clinico-biological significance. Haematologica. 2002;87(1):44-51.

22. Dohner H, Stilgenbauer S, Fischer K, Bentz M, Lichter P. Cytogenetic and molecular cytogenetic analysis of B cell chronic lymphocytic leukemia: Specific chromosome aberrations identify prognostic subgroups of patients and point to loci of candidate genes. Leukemia. 1997;11 Suppl 2:S19-S24.

23. Mato A, Nabhan C, Kay NE, et al. Realworld clinical experience in the Connect chronic lymphocytic leukaemia registry: a prospective cohort study of 1494 patients across 199 US centres. Br J Haematol. 2016;175(5):892-903

24. Sindelarova L, Michalova K, Zemanova Z, et al. Incidence of chromosomal anomalies detected with FISH and their clinical correlations in B-chronic lymphocytic leukemia. Cancer Genet Cytogenet. 2005;160(1):2734.

25. Strati P, Abruzzo LV, Wierda WG, O'Brien S, Ferrajoli A, Keating MJ. Second cancers and Richter transformation are the leading causes of death in patients with trisomy 12 chronic lymphocytic leukemia. Clin Lymphoma Myeloma Leuk. 2015;15(7):420427

26. Huh YO, Schweighofer CD, Ketterling RP, et al. Chronic lymphocytic leukemia with $t(14 ; 19)(q 32 ; q 13)$ is characterized by atypical morphologic and immunophenotypic features and distinctive genetic features. Am J Clin Pathol. 2011;135(5):686-696.

27. Sellmann L, Gesk S, Walter C, et al. Trisomy 19 is associated with trisomy 12 and mutated IGHV genes in B-chronic lymphocytic leukaemia. Br J Haematol. 2007;138(2):217220.

28. Daudignon A, Poulain S, Morel P, et al. Increased trisomy 12 frequency and a biased IgVH 3-21 gene usage characterize small lymphocytic lymphoma. Leuk Res.
2010;34(5):580-584.

29. Santos FP, O'Brien S. Small lymphocytic lymphoma and chronic lymphocytic leukemia: are they the same disease? Cancer J. 2012;18(5):396-403.

30. Lopez C, Delgado J, Costa D, et al. Different distribution of NOTCH1 mutations in chronic lymphocytic leukemia with isolated trisomy 12 or associated with other chromosomal alterations. Genes Chromosomes Cancer. 2012;51(9):881-889

31. Rossi D, Cerri M, Capello D, et al. Biological and clinical risk factors of chronic lymphocytic leukaemia transformation to Richter syndrome. Br J Haematol. 2008;142(2):202215.

32. Yi S, Li Z, Zou D, et al. Intratumoral genetic heterogeneity and number of cytogenetic aberrations provide additional prognostic significance in chronic lymphocytic leukemia. Genet Med. 2017;19(2):182-191

33. Falisi E, Novella E, Visco C, et al. B-cell receptor configuration and mutational analysis of patients with chronic lymphocytic leukaemia and trisomy 12 reveal recurrent molecular abnormalities. Hematol Oncol. 2014;32(1):22-30

34. Landau DA, Carter SL, Stojanov P, et al Evolution and impact of subclonal mutations in chronic lymphocytic leukemia. Cell. 2013;152(4):714-726.

35. Landau DA, Tausch E, Taylor-Weiner AN, et al. Mutations driving CLL and their evolution in progression and relapse. Nature. 2015;526(7574):525-530

36. Zent CS, Ding W, Schwager SM, et al. The prognostic significance of cytopenia in chronic lymphocytic leukaemia/small lymphocytic lymphoma. Br J Haematol. 2008;141(5):615-621

37. Strati P, Caligaris-Cappio F. A matter of debate in chronic lymphocytic leukemia: is the occurrence of autoimmune disorders an indicator of chronic lymphocytic leukemia therapy? Curr Opin Oncol. 2011;23(5):455460.

38. Athanasiadou A, Stamatopoulos $\mathrm{K}$, Tsompanakou A, et al. Clinical, immunophenotypic, and molecular profiling of trisomy 12 in chronic lymphocytic leukemia and comparison with other karyotypic subgroups defined by cytogenetic analysis. Cancer Genet Cytogenet. 2006;168 (2):109-119.

39. Matutes E, Oscier D, Garcia-Marco J, et al. Trisomy 12 defines a group of CLL with atypical morphology: Correlation between cytogenetic, clinical and laboratory features in 544 patients. Br J Haematol. 1996;92(2): 382-388.

40. Nowakowski GS, Hoyer JD, Shanafelt TD, et al. Percentage of smudge cells on routine blood smear predicts survival in chronic lymphocytic leukemia. J Clin Oncol. 2009;27(11):1844-1849.

41. Saunders AE, Johnson P. Modulation of immune cell signalling by the leukocyte common tyrosine phosphatase, CD45. Cell Signal. 2010;22(3):339-348.

42. Johansson P, Eisele L, Klein-Hitpass L, et al. Percentage of smudge cells determined on routine blood smears is a novel prognostic factor in chronic lymphocytic leukemia. Leuk Res. 2010;34(7):892-898.

43. Rizzo D, Lotay A, Gachard N, et al. Very low levels of surface CD45 reflect CLL cell fragility, are inversely correlated with trisomy 12 and are associated with increased treatment-free survival. Am J Hematol. 2013:88(9):747-753

44. Cro L, Ferrario A, Lionetti M, et al. The clin- ical and biological features of a series of immunophenotypic variant of B-CLL. Eur J Haematol. 2010;85(2):120-129.

45. Deaglio S, Morra M, Mallone $\mathrm{R}$, et al Human CD38 (ADP-ribosyl cyclase) is a counter-receptor of CD31, an Ig superfamily member. J Immunol. 1998;160(1):395-402.

46. Riches JC, O'Donovan CJ, Kingdon SJ, et al. Trisomy 12 chronic lymphocytic leukemia cells exhibit upregulation of integrin signaling that is modulated by NOTCH1 mutations. Blood. 2014;123(26):4101-4110.

47. Moreau C, Liu Q, Graeff R, et al. CD38 structure-based inhibitor design using the 1 cyclic inosine 50-diphosphate ribose template. PLoS One. 2013;8(6):e66247.

48. Damle RN, Wasil T, Fais F, et al. Ig V gene mutation status and CD38 expression as novel prognostic indicators in chronic lymphocytic leukemia. Blood. 1999;94(6):18401847

49. Hamblin TJ, Orchard JA, Ibbotson RE, et al. CD38 expression and immunoglobulin variable region mutations are independent prognostic variables in chronic lymphocytic leukemia, but CD38 expression may vary during the course of the disease. Blood. 2002;99(3):1023-1029.

50. Jelinek DF, Tschumper RC, Geyer SM, et al Analysis of clonal B-cell CD38 and immunoglobulin variable region sequence status in relation to clinical outcome for Bchronic lymphocytic leukaemia. Br J Haematol. 2001:115(4):854-861.

51. D'Arena G, Musto P, Cascavilla N, et al CD38 expression correlates with adverse biological features and predicts poor clinical outcome in B-cell chronic lymphocytic leukemia. Leuk Lymphoma. 2001;42(12):109-114.

52. Brachtl G, Pinon Hofbauer J, Greil R, Hartmann TN. The pathogenic relevance of the prognostic markers CD38 and CD49d in chronic lymphocytic leukemia. Ann Hematol. 2014;93(3):361-374.

53. Wiestner A, Rosenwald A, Barry TS, et al. ZAP-70 expression identifies a chronic lymphocytic leukemia subtype with unmutated immunoglobulin genes, inferior clinical outcome, and distinct gene expression profile. Blood. 2003;101(12):4944-4951

54. Crespo M, Bosch F, Villamor N, et al. ZAP70 expression as a surrogate for immunoglobulin-variable-region mutations in chronic lymphocytic leukemia. N Engl J Med. 2003;348(18):1764-1775

55. Tam CS, Otero-Palacios J, Abruzzo LV, et al. Chronic lymphocytic leukaemia CD20 expression is dependent on the genetic subtype: a study of quantitative flow cytometry and fluorescent in-situ hybridization in 510 patients. Br J Haematol. 2008;141(1):36-40.

56. Gattei V, Bulian P, Del Principe MI, et al. Relevance of CD49d protein expression as overall survival and progressive disease prognosticator in chronic lymphocytic leukemia. Blood. 2008:111(2):865-873.

57. Shanafelt TD, Geyer SM, Bone ND, et al. CD49d expression is an independent predictor of overall survival in patients with chronic lymphocytic leukaemia: a prognostic parameter with therapeutic potential. Br J Haematol. 2008;140(5):537-546.

58. Dal Bo M, Bomben R, Zucchetto A, et al. Microenvironmental interactions in chronic lymphocytic leukemia: hints for pathogenesis and identification of targets for rational therapy. Curr Pharm Des. 2012;18(23):33233334.

59. Zucchetto A, Caldana C, Benedetti D, et al. CD49d is overexpressed by trisomy 12 
chronic lymphocytic leukemia cells: evidence for a methylation-dependent regulation mechanism. Blood. 2013;122(19):33173321.

60. Strati P, Parikh SA, Chaffee KG, et al. CD49d associates with nodal presentation and subsequent development of lymphadenopathy in patients with chronic lymphocytic leukaemia. Br J Haematol. 2017;178(1):99105.

61. Shanafelt TD, Hanson C, Dewald GW, et al. Karyotype evolution on fluorescent in situ hybridization analysis is associated with short survival in patients with chronic lymphocytic leukemia and is related to CD49d expression. J Clin Oncol. 2008;26(14):e5-e6.

62. Conrad DH, Ford JW, Sturgill JL, Gibb DR. CD23: an overlooked regulator of allergic disease. Curr Allergy Asthma Rep. 2007;7(5):331-337.

63. Barna G, Reiniger L, Tatrai P, Kopper L, Matolcsy A. The cutoff levels of CD23 expression in the differential diagnosis of MCL and CLL. Hematol Oncol. 2008;26 (3):167-170

64. Jurisic V, Colovic N, Kraguljac N, Atkinson $\mathrm{HD}$, Colovic M. Analysis of CD23 antigen expression in B-chronic lymphocytic leukaemia and its correlation with clinical parameters. Med Oncol. 2008;25(3):315-322.

65. Fournier S, Yang LP, Delespesse G, Rubio M, Biron G, Sarfati M. The two CD23 isoforms display differential regulation in chronic lymphocytic leukaemia. Br J Haematol. 1995;89(2):373-379.

66. Kriston C, Bödör C, Szenthe K, et al. Low CD23 expression correlates with high CD38 expression and the presence of trisomy 12 in CLL. Hematol Oncol. 2017;35(1):58-63.

67. Burton J, Kay NE. Does IL-2 receptor expression and secretion in chronic B-cell leukemia have a role in downregulation of the immune system? Leukemia. 1994;8(1):92-96.

68. Molica S, Levato D, Dell'Olio M, et al. Clinico-prognostic implications of increased levels of soluble CD54 in the serum of B-cell chronic lymphocytic leukemia patients. Results of a multivariate survival analysis. Haematologica. 1997;82(2):148-151.

69. Lúcio PJ, Faria MT, Pinto AM, et al. Expression of adhesion molecules in chronic B-cell lymphoproliferative disorders. Haematologica. 1998;83(2):104-111.

70. Angelopoulou MK, Kontopidou FN, Pangalis GA. Adhesion molecules in Bchronic lymphoproliferative disorders. Semin Hematol. 1999;36(2):178-197.

71. Hjalmar V, Hast R, Kimby E. Cell surface expression of CD25, CD54, and CD95 on Band T-cells in chronic lymphocytic leukaemia in relation to trisomy 12 , atypical morphology and clinical course. Eur J Haematol. 2002;68(3):127-134.

72. Tefferi A, Bartholmai B, Witzig TE, et al. Clinical correlations of immunophenotypic variations and the presence of trisomy 12 in B-cell chronic lymphocytic leukemia. Cancer Genet Cytogenet. 1997;95(2):173-177.

73. Knauf WU, Knuutila S, Zeigmeister B, Thiel
E. Trisomy 12 in B-cell chronic lymphocytic leukemia: Correlation with advanced disease, atypical morphology, high levels of soluble CD25, and with refractoriness to treatment. Leuk Lymphoma. 1995;19(3-4):289294.

74. Knauf WU, Langenmayer I, Ehlers B, et al. Serum levels of soluble CD23, but not soluble $\mathrm{CD} 25$, predict disease progression in early stage B-cell chronic lymphocytic leukemia. Leuk Lymphoma. 1997;89(11): 4241-4242

75. Darzentas N, Hadzidimitriou A, Murray F, et al. A different ontogenesis for chronic lymphocytic leukemia cases carrying stereotyped antigen receptors: molecular and computational evidence. Leukemia. 2010;24(1): 125-132.

76. Rosén A, Murray F, Evaldsson C, Rosenquist R. Antigens in chronic lymphocytic leukemia-implications for cell origin and leukemogenesis. Semin Cancer Biol. 2010;20(6):400-409.

77. Maura F, Cutrona G, Fabris S, et al. Relevance of stereotyped B-cell receptors in the context of the molecular, cytogenetic and clinical features of chronic lymphocytic leukemia. PLoS One. 2011;6(8):e24313.

78. Rossi D, Spina V, Cerri M, et al. Stereotyped $\mathrm{B}$-cell receptor is an independent risk factor of chronic lymphocytic leukemia transformation to Richter syndrome. Clin Cancer Res. 2009;15(13):4415-4422.

79. Stamatopoulos K, Belessi C, Moreno C, et al. Over $20 \%$ of patients with chronic lymphocytic leukemia carry stereotyped receptors: pathogenetic implications and clinical correlations. Blood. 2007;109(1):259-270.

80. Athanasiadou A, Stamatopoulos K, Gaitatzi M, Stavroyianni N, Fassas A, Anagnostopoulos A. Recurrent cytogenetic findings in subsets of patients with chronic lymphocytic leukemia expressing IgGswitched stereotyped immunoglobulins. Haematologica. 2008;93(3):473-474

81. Zenz T, Vollmer D, Trbusek M, et al. TP53 mutation profile in chronic lymphocytic leukemia: evidence for a disease specific profile from a comprehensive analysis of 268 mutations. Leukemia. 2010;24(12):2072 2079.

82. González-Gascón y Marín I, HernándezSánchez M, Rodríguez-Vicente AE, et al. A high proportion of cells carrying trisomy 12 is associated with a worse outcome in patients with chronic lymphocytic leukemia. Hematol Oncol. 2016:34(2):84-92.

83. Villamor N, Conde L, Martínez-Trillos A, et al. NOTCH1 mutations identify a genetic subgroup of chronic lymphocytic leukemia patients with high risk of transformation and poor outcome Leukemia. 2013;27(5):1100-1106.

84. Balatti V, Bottoni A, Palamarchuk A, et al. NOTCH1 mutations in CLL associated with trisomy 12. Blood. 2012;119(2):329-331.

85. Balatti V, Lerner S, Rizzotto L, et al. Trisomy 12 CLLs progress through NOTCH1 mutations. Leukemia. 2013;27(3):740-743.
86. Weissmann S, Roller A, Jeromin S, et al. Prognostic impact and landscape of NOTCH1 mutations in chronic lymphocytic leukemia (CLL): a study on 852 patients. Leukemia. 2013;27(12):2393-2396.

87. Sportoletti P, Baldoni S, Cavalli L, et al. NOTCH1 PEST domain mutation is an adverse prognostic factor in B-CLL. Br J Haematol. 2010;151(4):404-406.

88. Fabbri G, Rasi S, Rossi D, et al. Analysis of the chronic lymphocytic leukemia coding genome: role of NOTCH1 mutational activation. J Exp Med. 2011;208(7):1389-1401.

89. Puente XS, Pinyol M, Quesada V, et al. Whole genome sequencing identifies recurrent mutations in chronic lymphocytic leukaemia. Nature. 2011;475(7354):101-105.

90. Oscier DG, Rose-Zerilli MJ, Winkelmann N, et al. The clinical significance of NOTCH1 and SF3B1 mutations in the UK LRF CLL4 trial. Blood. 2013;121(3):468-475.

91. Del Giudice I, Rossi D, Chiaretti S, et al. NOTCH1 mutations in +12 chronic lymphocytic leukemia (CLL) confer an unfavorable prognosis, induce a distinctive transcriptional profiling and refine the intermediate prognosis of +12 CLL. Haematologica. 2012;97(3):437-441.

92. Rossi D, Rasi S, Fabbri G, et al. Mutations of NOTCH1 are an independent predictor of survival in chronic lymphocytic leukemia. Blood. 2012;119(2):521-529.

93. Rossi D, Spina V, Deambrogi C, et al. The genetics of Richter syndrome reveals disease heterogeneity and predicts survival after transformation. Blood. 2011;117(12):33913401.

94. Yaktapour N, Übelhart R, Schüler J, et al. Insulin-like growth factor-1 receptor (IGF1R) as a novel target in chronic lymphocytic leukemia. Blood. 2013;122(9):1621-1633.

95. Maura F, Mosca L, Fabris S, et al. Insulin growth factor 1 receptor expression is associated with NOTCH1 mutation, trisomy 12 and aggressive clinical course in chronic lymphocytic leukaemia. PLoS One. 2015;10 (3): e0118801.

96. Medyouf $\mathrm{H}$, Gusscott $\mathrm{S}$, Wang $\mathrm{H}$, et al High-level IGF1R expression is required for leukemia-initiating cell activity in T-ALL and is supported by Notch signaling. J Exp Med. 2011:208(9):1809-1822.

97. Mittal AK, Hegde GV, Aoun P, et al. Molecular basis of aggressive disease in chronic lymphocytic leukemia patients with 11q deletion and trisomy 12 chromosomal abnormalities. Int J Mol Med. 2007;20(4): 461-469.

98. Tsimberidou AM, Wen S, McLaughlin P, et al. Other malignancies in chronic lymphocytic leukemia/small lymphocytic lymphoma. J Clin Oncol. 2009;27(6):904-910.

99. Solomon BM, Rabe KG, Slager SL, Brewer JD, Cerhan JR, Shanafelt TD. Overall and cancer-specific survival of patients with breast, colon, kidney, and lung cancers with and without chronic lymphocytic leukemia: a SEER population-based study. J Clin Oncol. 2013;31(7):930-937. 